\title{
Characterizing the Access of Clinical Decision Support Offered by Immunization Information System in Minnesota
}

\author{
Sripriya Rajamani ${ }^{1 *}$, Aaron Bieringer ${ }^{2}$, Miriam Muscoplat ${ }^{2}$ \\ ${ }^{1}$ Public Health Informatics Program, School of Public Health, University of Minnesota, Minneapolis, \\ Minnesota \\ ${ }^{2}$ Minnesota Immunization Information Connection (MIIC), Immunization Program, Minnesota \\ Department of Health, St. Paul, Minnesota
}

\begin{abstract}
Background: Healthy People 2020 aims to improve population health by increasing immunization rates to decrease vaccine-preventable infectious diseases. Amongst the many strategies, role of immunization information systems (IIS) are recognized by studies and taskforce reports. IIS are unique in their offering of clinical decision support for immunizations (CDSi) which are utilized by healthcare providers. Federal initiatives such as Meaningful Use (MU) and Affordable Care Act (ACA) aim to improve immunization rates through use of technology and expanding access to immunization services respectively. MU, the Electronic Health Record (EHR) incentive program includes use of IIS CDSi functionality as part of Stage 3. It is essential to understand access and use patterns of IIS CDSi, so as to utilize it better to improve immunization services.
\end{abstract}

Objectives: To understand the utilization of clinical decision support for immunizations (CDSi) offered by immunization information system in Minnesota and to analyze the variability of its use across providers and EHR implementations.

Methods: IIS in Minnesota (Minnesota Immunization Information Connection: MIIC) offers CDSi that is accessed through EHRs and branded as Alternate Access (AA). Data from MIIC and technical documents were reviewed to create details on organizations which implemented AA functionality. Data on EHR adoption in clinics and local health departments was obtained from Minnesota eHealth assessment reports. Data on access were tracked from January 2015 through mid-October 2015 through weekly specialized reports to track the queries by organization, volume and day of the week. Data were analyzed, findings were synthesized and reviewed with subject matter experts.

Conclusion: High EHR adoption offers a great opportunity to promote use of IIS CDSi at point of care. Analysis did not track use at individual clinic level and how the queries were being generated. Additional research is needed to understand the provider level use of this CDSi and other organizational and technical factors which influence access to IIS. This is essential for IIS as they execute projects to improve population-level immunization rates, plan provider outreach and prioritize their system enhancements to meet federal requirements.

Keywords: clinical decision support, immunizations, public health informatics, bidirectional, immunization information systems (IIS), electronic health records (EHRs) 
DOI: 10.5210/ojphi.v7i3.6282

Copyright $@ 2015$ the author(s)

This is an Open Access article. Authors own copyright of their articles appearing in the Online Journal of Public Health Informatics. Readers may copy articles without permission of the copyright owner(s), as long as the author and OJPHI are acknowledged in the copy and the copy is used for educational, not-for-profit purposes.

\section{Introduction}

Immunizations are proven to be the key success factor for the decline of many infectious diseases and are recognized as a highly cost-effective clinical preventive service [1]. One of the goals of the Healthy People 2020 which seeks to improve population health is to increase immunization rates and improve proper immunization series compliance to reduce preventable infectious diseases [1]. Amongst the many strategies to improve immunization compliance, adoption of Immunization Information Systems (IIS) is increasingly recognized by various studies and taskforce reports as a key and effective tactic [2]. IIS are population-based, confidential computerized systems present in most US states and territories [3]. IIS have the unique advantage of holding immunization data across various providers over time, therefore offering comprehensive vaccination histories.

The vision for management of immunization information is availability of real-time, consolidated data and services for all ages, to clinical, public health and other stakeholders within their workflow [3]. One of the modalities for achieving this vision is through Immunization Information System (IIS). The IIS in Minnesota, known as Minnesota Immunization Information Connection (MIIC) [4] has been in operation for more than 12 years. MIIC contains 75,855,190 million immunization records for 7,675,687 people, as of October 2015. Minnesota's healthcare landscape is unique with high electronic health record (EHR) adoption supported by state initiatives such as Interoperable EHR mandate [5], state health reform [6], state innovation model grant efforts [7] and federal incentives for EHRs, referred to as Meaningful Use [8].

Recommendations on vaccines from the Advisory Committee on Immunization Practices (ACIP) [9] are disseminated through various modalities including IIS. IIS contain vaccine forecasting algorithms to predict immunizations, also known as clinical decision support for immunizations (CDSi). This evaluation and forecasting is complex, including factors like age for vaccine administration, number of doses, their intervals, precautions, and contraindications. With increase in use of EHRs, some of these complex CDSi rules have been built into EHRs as CDS modules and/or accessed from IIS (through EHRs or directly via IIS interface). Due to immunization schedule complexity, the need for comprehensive vaccination history for accurate predictions, and the variability of CDSi across provider groups and across EHR implementations, it is recommended to access and use the CDSi from IIS. IIS serve as a powerful tool for improving vaccination rates [2] and CDSi in IIS play an important role.

Bi-directional communication between clinical and public health is an emerging field with previous work focusing primarily on clinician alerts for diseases [10,11]. Study on access to IIS decision support within EHR [12] concluded that visual integration of external registries into an 
EHR was feasible with improvement in provider satisfaction and registry reporting. Study by Rajamani et al. [13] on submission of data to MIIC highlighted uptake of standards-based reporting in the last three years. These studies point to increasing technical capacities of provider organizations, and serve as a barometer to their capabilities for implementing new functionalities. Research by Stockwell et al. [14] evaluated immunization data exchange between EHR and IIS for informing Stage $3 \mathrm{MU}$ and outlined its benefits. Bi-directionality across IIS and EHRs has been advocated wherein benefits of integration supersede the challenges posed by variability in EHR technologies and various IIS [15]. The overlap of functionalities between EHRs and IIS have been outlined with recommendations for guiding their integration [16].

MIIC currently offers an option branded as 'Alternate Access' (AA) [4] to access MIIC and the CDSi from within the provider EHR. This solution offers the ability to generate a query to MIIC for vaccination history and forecasting and is based on demographics of the record in EHR. This option addresses the issue of repeat data entry for the query and also does not require logging into the MIIC system separately. With this AA functionality, the history and forecast are presented to user within their EHR. This data can either be displayed as 'read-only' view or can be 'integrated' and most have implemented 'read-only' option. The current response for queries to access MIIC CDSi is custom-built, but will be updated to recommended standards.

Meaningful Use (MU), the Electronic Health Record (EHR) incentive program recognizes public and population health as one of the priority areas [8]. It focuses on tactics using health information technology to achieve public health objectives. Stage $1 \mathrm{MU}$ recommended reporting to public health including IIS, based on nationally recognized standards. Stage 3 MU [17] has drawn to the CDSi functionality of IIS by recommending bi-directional communications between certified EHR technology and IIS which enables submission of immunization data and receiving immunization forecasts and histories from IIS. Affordable Care Act (ACA) expands and strengthens access to immunization services for children and adults [18]. ACA also fastens the time for availability and coverage of ACIP recommended vaccines. IIS CDSi which incorporates ACIP recommendations and has vaccine forecasting based on comprehensive vaccination history in IIS is a great tool to promote immunizations at point of care.

There is a need to understand the current status of adoption of IIS CDSi functionality, its access and use patterns (profile of users, their volume of queries and the number of records found), so as to utilize it better to improve immunization services. The study objectives are to understand the utilization of IIS CDSi through EHR access option, a feature offered by IIS in Minnesota (MIIC) and to analyze the variability of its use across providers and EHR implementations.

\section{Methods}

Minnesota Immunization Information Connection (MIIC), the IIS for the state of Minnesota has 4,852 organizations which participate in the IIS currently. Some of these organizations submit data for reporting purposes (e.g., health systems including clinics, hospitals) and some have access to data which are read-only (e.g., schools). All organizations can use the IIS interface to access the clinical decision support (CDSi) functionality offered by MIIC. A sub-set of submitting organizations have the ability to access MIIC from their EHRs through their implementations of Alternate Access functionality. Data from various sources and customized queries were compiled, analyzed and reviewed with subject matter experts. The study framework is depicted in Figure 1 and outlines the sources and approach to characterize the overall utilization of MIIC CDSi. 


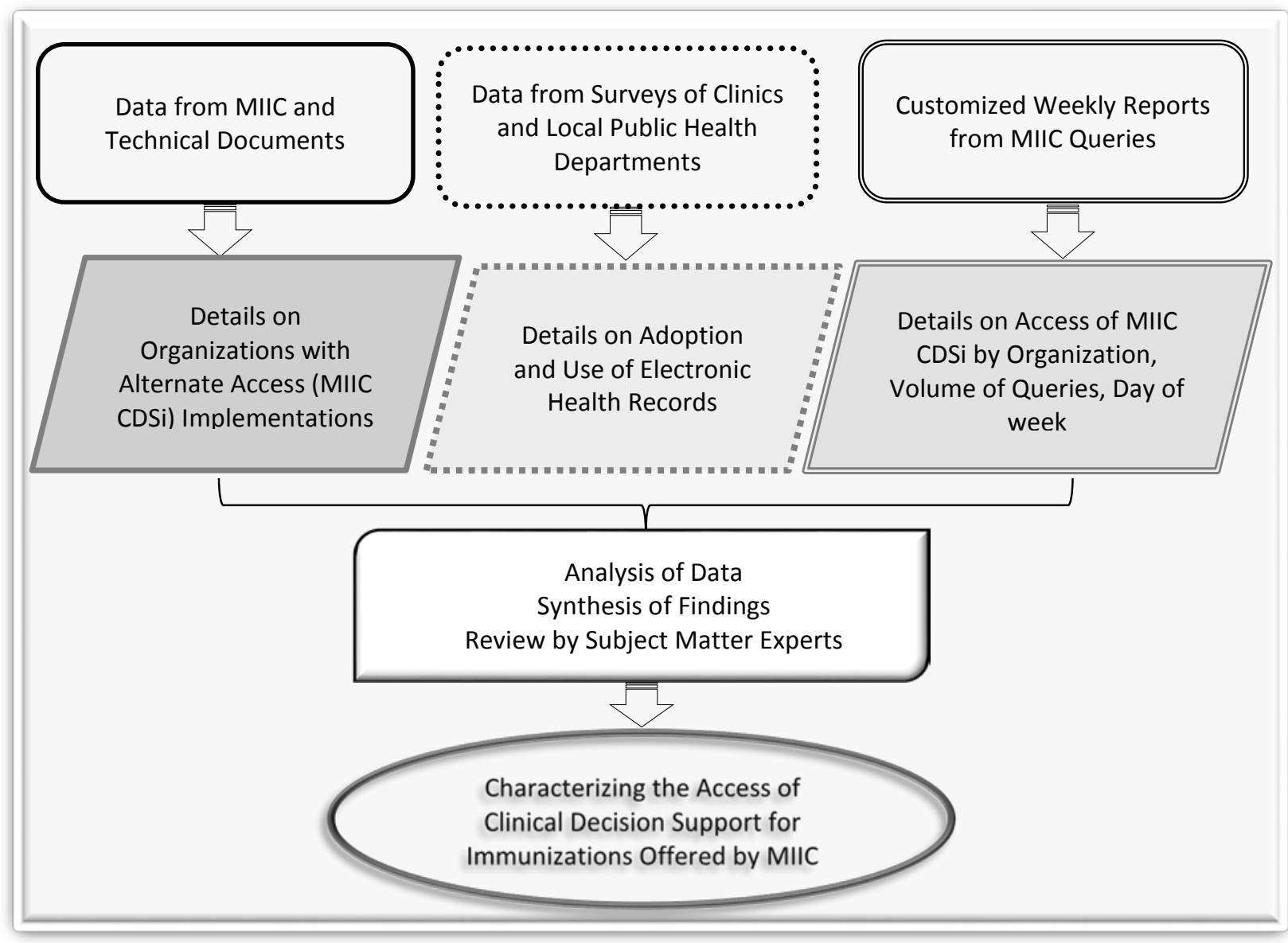

Figure 1: Study Framework

The framework to characterize MIIC CDSi access comprised of: (1) healthcare organizations, number of affiliated sites, EHR platform; (2) trend in queries for January through September 2015 and stratified by healthcare system vs. local public health; and (3) detailed analysis of records found for healthcare system queries by organizations and for one week period October $12-18$, 2015. Results were discussed with subject matter experts to validate findings and to gather contextual information about the organization and any additional data on MIIC CDSi use.

\section{Results}

Minnesota has high rates of EHR adoption in clinics with nearly all (97\%) ambulatory clinics with EHRs, representing 1,146 clinics in 2014. Epic is the dominant EHR vendor system and is in use by $49 \%$ of Minnesota's clinics which have adopted EHRs (Table 1). Table 2 presents the EHR adoption and vendor data for local public health for the year 2014 and all reported use of EHRs with the exception of two local public health departments. PH-Doc product was the prevalent EHR, used by 28 out of 50 (56\%) local health departments. 
Table 1: EHR System Adoption and Vendors in Minnesota Clinics, 2014

\begin{tabular}{|c|c|c|}
\hline Electronic Health Record Systems & Percent & Count \\
\hline Epic & $49 \%$ & 559 \\
\hline NextGen & $6 \%$ & 71 \\
\hline Allscripts & $6 \%$ & 70 \\
\hline eClinicalWorks & $6 \%$ & 67 \\
\hline Cerner & $4 \%$ & 50 \\
\hline Centricity & $4 \%$ & 45 \\
\hline Greenway & $3 \%$ & 40 \\
\hline Meditech & $2 \%$ & 24 \\
\hline Other & $19 \%$ & 220 \\
\hline Total & & 1,146 \\
\hline
\end{tabular}

Table 2: EHR Adoption and Vendors in Minnesota Local Public Health Departments*, 2014

\begin{tabular}{lll}
\hline Electronic Health Record Systems & Percent & Count \\
\hline PH-Doc & $\mathbf{5 6 \%}$ & $\mathbf{2 8}$ \\
CHAMP & $36 \%$ & 18 \\
CareFacts & $12 \%$ & 6 \\
Custom-built local system & $8 \%$ & 4 \\
Digital Health Department & $4 \%$ & 2 \\
Decade & $2 \%$ & 1 \\
Other & $10 \%$ & 5 \\
Total & & $50^{*}$ \\
\hline${ }^{*}$ Assessed at Community Health Board (CHB) level; more than 1 system may be used in \\
\hline
\end{tabular}

Table 3 presents the organizations that have implemented the AA functionality and number of affiliated sites with access and currently this comprises of 25 healthcare systems/organizations representing 599 individual provider sites. The number of sites accessing an AA installation varied from 1 to as much as 110 affiliated sites. Healthcare systems have the most sites (559 out of 599) with access to this functionality. With the exception of one local public health system with 23 affiliated sites, most were single installations. 
Table 3: Organizations with MIIC CDSi Implementations: Affiliated Sites, EHR Systems

\begin{tabular}{|c|c|c|}
\hline Organization & $\begin{array}{c}\text { Number of } \\
\text { Affiliated Sites Using } \\
\text { Functionality }\end{array}$ & EHR System \\
\hline \multicolumn{3}{|l|}{ Healthcare Systems } \\
\hline Health System A & 110 & Epic \\
\hline Health System B & 62 & Epic \\
\hline Health System C & 12 & Cerner \\
\hline Health System D & 83 & Epic \\
\hline Health System E & 93 & Epic \\
\hline Health System F & 4 & Epic \\
\hline Health System G & 84 & Epic \\
\hline Health System H & 28 & Epic \\
\hline Health System I & 83 & GE Centricity (transitioning to Epic) \\
\hline \multicolumn{3}{|c|}{ Local Public Health Departments } \\
\hline Local Public Health A & 1 & PH-Doc \\
\hline Local Public Health B & 1 & PH-Doc \\
\hline Local Public Health C & 1 & PH-Doc \\
\hline Local Public Health D & 2 & PH-Doc \\
\hline Local Public Health E & 1 & PH-Doc \\
\hline Local Public Health F & 1 & PH-Doc \\
\hline Local Public Health G & 1 & PH-Doc \\
\hline Local Public Health H & 23 & PH-Doc \\
\hline Local Public Health I & 1 & PH-Doc \\
\hline Local Public Health J & 2 & PH-Doc \\
\hline Local Public Health K & 1 & PH-Doc \\
\hline Local Public Health L & 1 & PH-Doc \\
\hline Local Public Health M & 1 & PH-Doc \\
\hline Local Public Health N & 1 & PH-Doc \\
\hline Local Public Health O & 1 & PH-Doc \\
\hline Local Public Health P & 1 & PH-Doc \\
\hline
\end{tabular}


Figure 2 displays the analysis of weekly Alternate Access reports to understand the volume of queries over a nine-month period from January to September 2015. The query count of health care systems (labeled Health Care System A - H) ranged from 1000 to 12,000 per week whereas Health System I had query count in ranges of 30,000 - 40,000 per week. This health system I was an outlier in terms of its access of AA functionality with exceedingly large number of queries. This query volume could not be explained with current metric tracking and requires additional information to understand pattern of access and use. The overall access volume didn't vary across weeks/months. Health System I had high volume in July which exceeded 300,000 queries to MIIC.

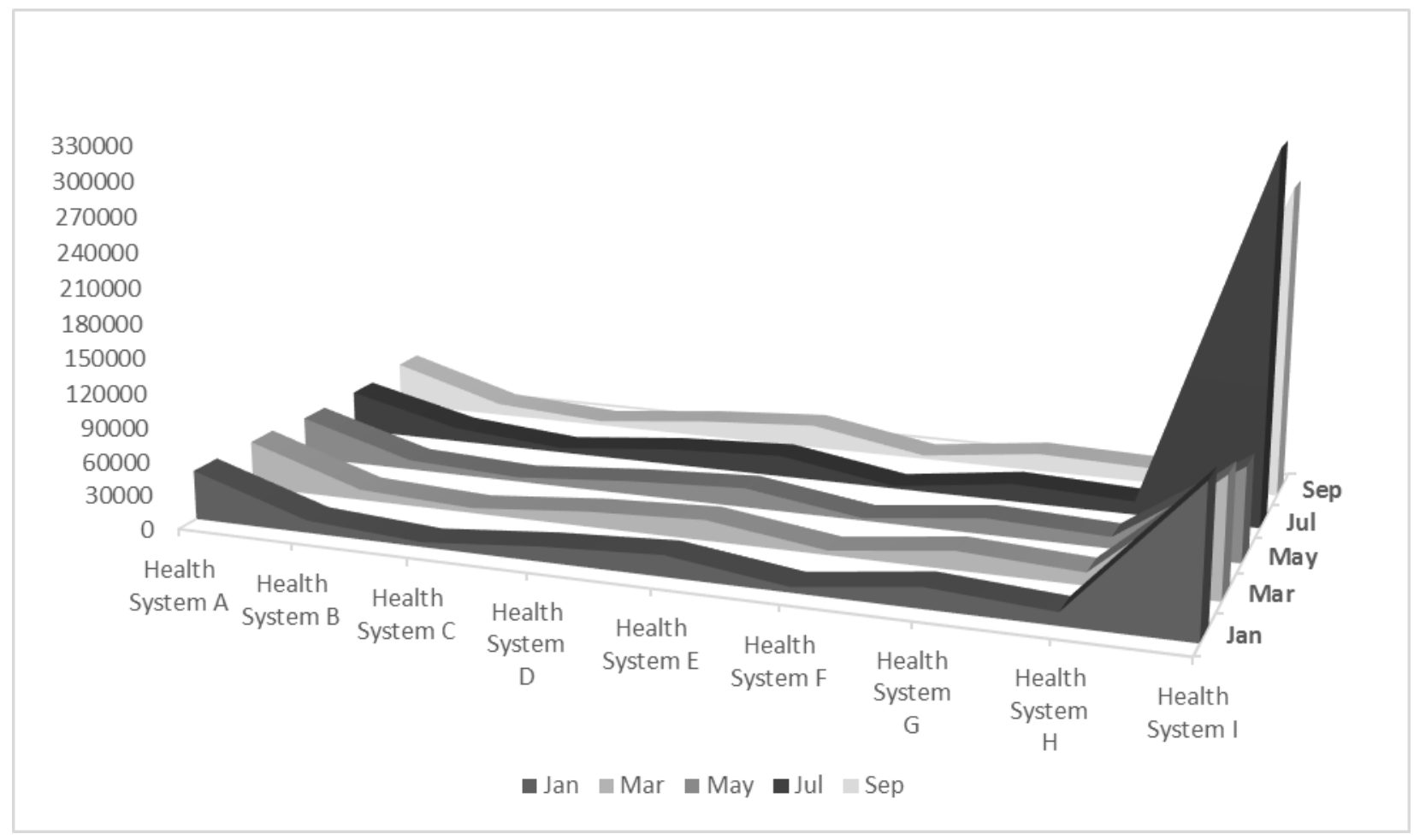

Figure 2: Queries to Access MIIC CDSi by Health Systems, January-September 2015

Figures 3 and 4 present the details of analysis of queries in week of 10/12/15 to 10/18/15 and is stratified by health system and local public health respectively. The queries in this week range from 11,625 (Health System A), 3,621 (Health System B), 1,776 (Health System C), 3,395 (Health System D), 5,829 (Health System E), 995 (Health System F), 4,336 (Health System G) and 3,076 (Health System H). Figure 3 excludes Health System I which was an outlier with 73,659 queries. For the week of 10/12/15 to 10/18/15, the query volume by local public health was in the range of 50 or less, with the exception of one system I which had 581 queries to access MIIC CDSi. Local public health systems $\mathrm{P}$ and $\mathrm{F}$ were next highest users with queries in range of 60. Local public health system I has implemented AA functionality for many years and involved in public health information exchange projects and that could attribute to the increased volume of queries. 


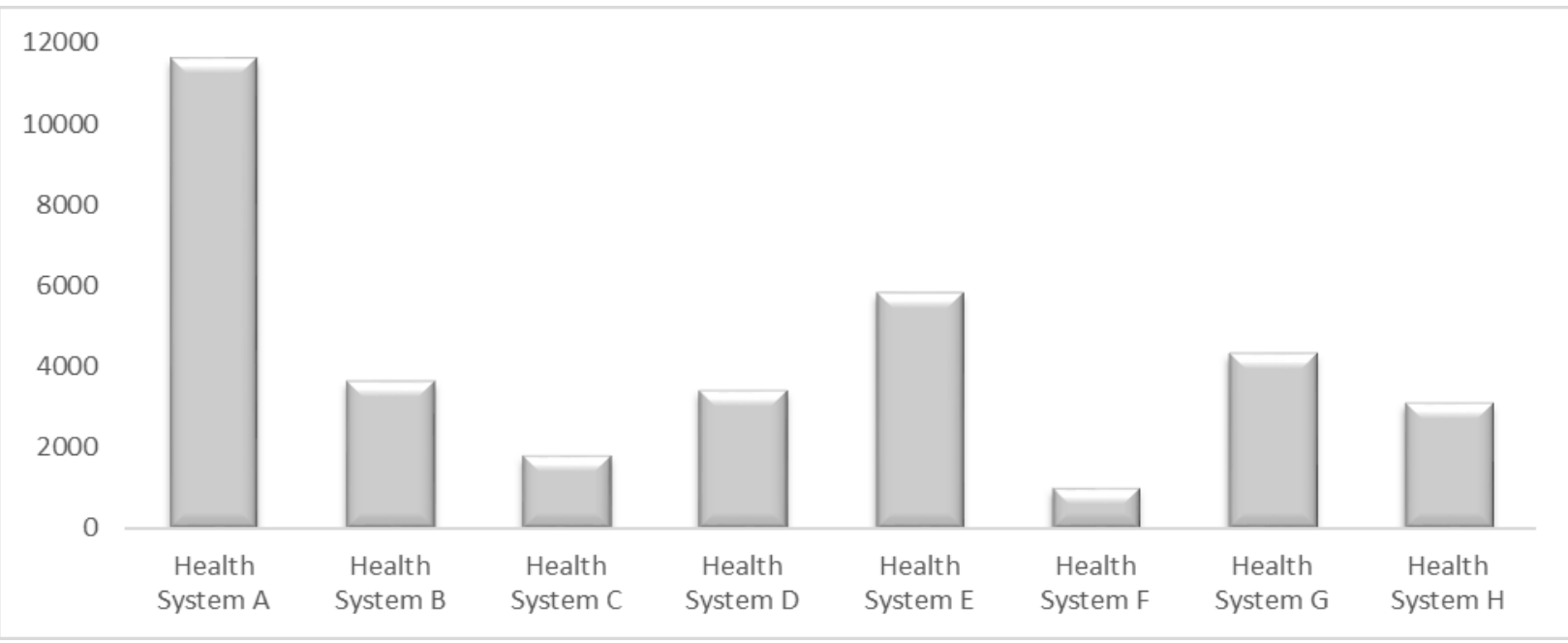

Figure 3: Queries to Access MIIC CDSi by Health Systems*, Week of 10/12/15 - 10/18/15 *Graph does not include data from Health System I, an outlier with much higher volume of queries

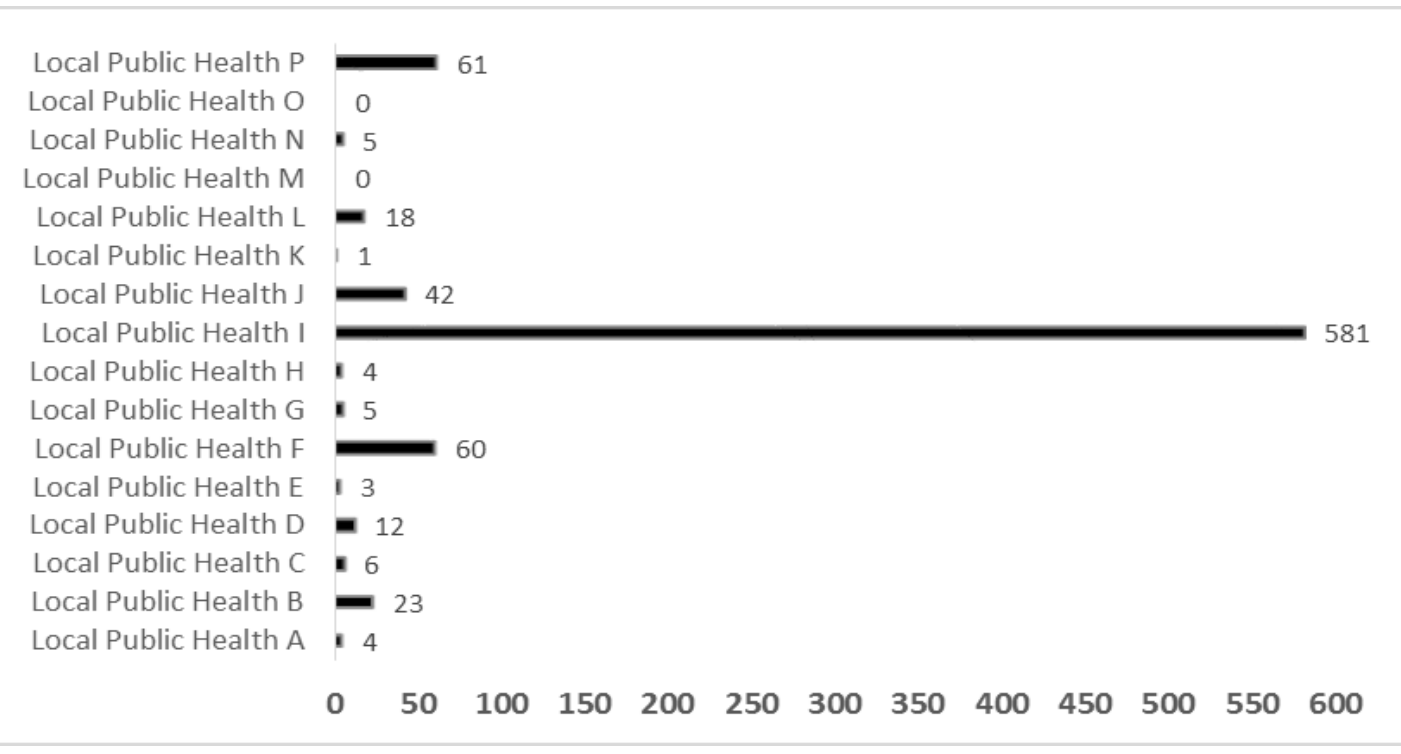

Figure 4: Queries to Access MIIC CDSi by Local Public Health, 10/12/15 - 10/18/15

The number of records located through Alternate Access was analyzed in detail for the week of 10/12/15 through 10/18/15. Figure 5 depicts that query was able to successfully locate a single matching record for 59\% of searches, could not find a record for $5 \%$ and resulted in blank/error for $31 \%$ of queries. Figure 6 presents the varying match rates across health systems with some searches yielding no records to some searches resulting in 13 records. Similar variability was found across local public health systems as well (Table 4), but the maximum records found in a search was six. Figure 7 shows that the volume of queries to access CDSi was less on week-ends (Saturday and Sunday), when compared to week days (Monday through Friday). 


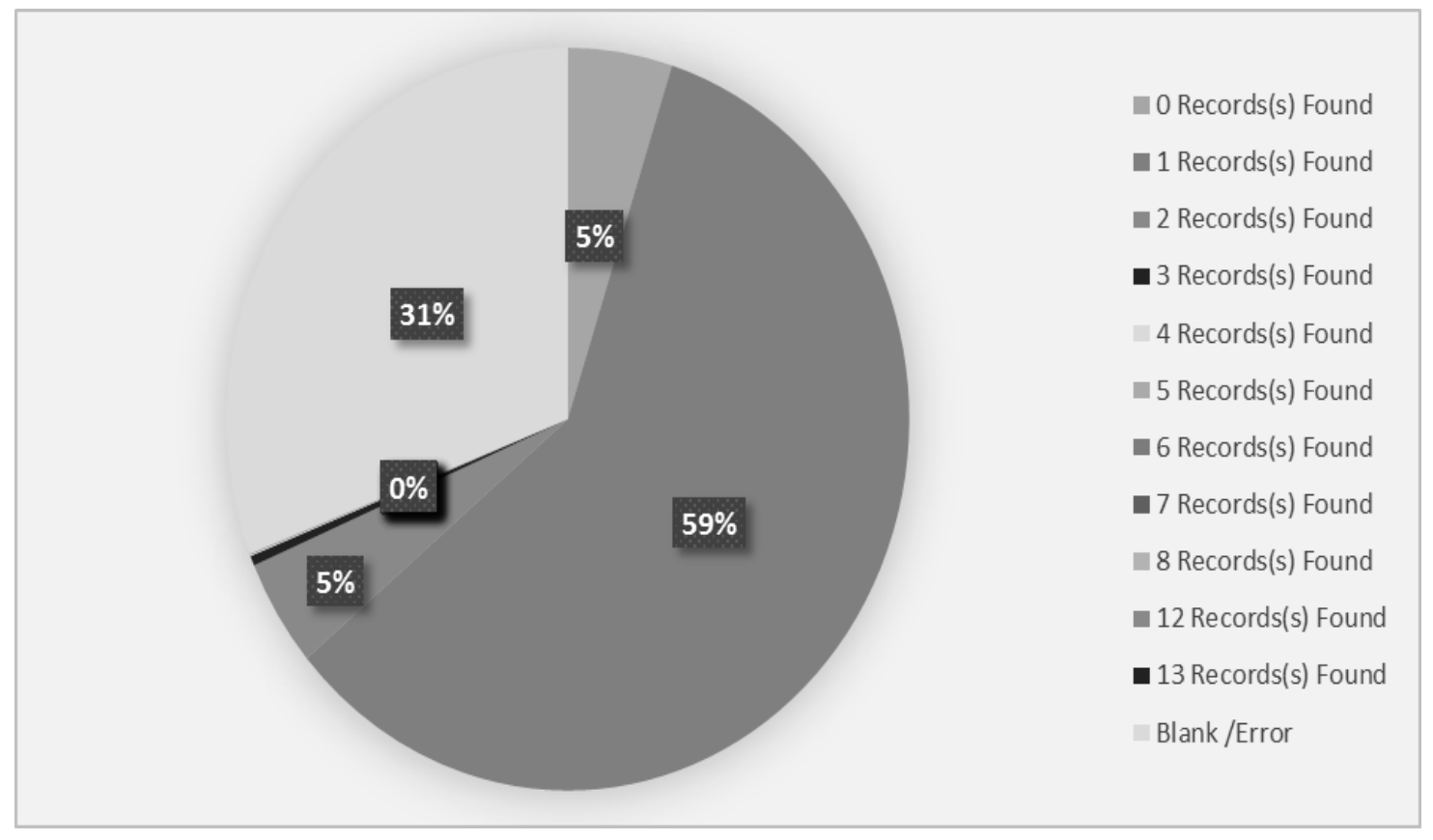

Figure 5: MIIC CDSi Query Results by Records Found, Week of 10/12/15 - 10/18/15

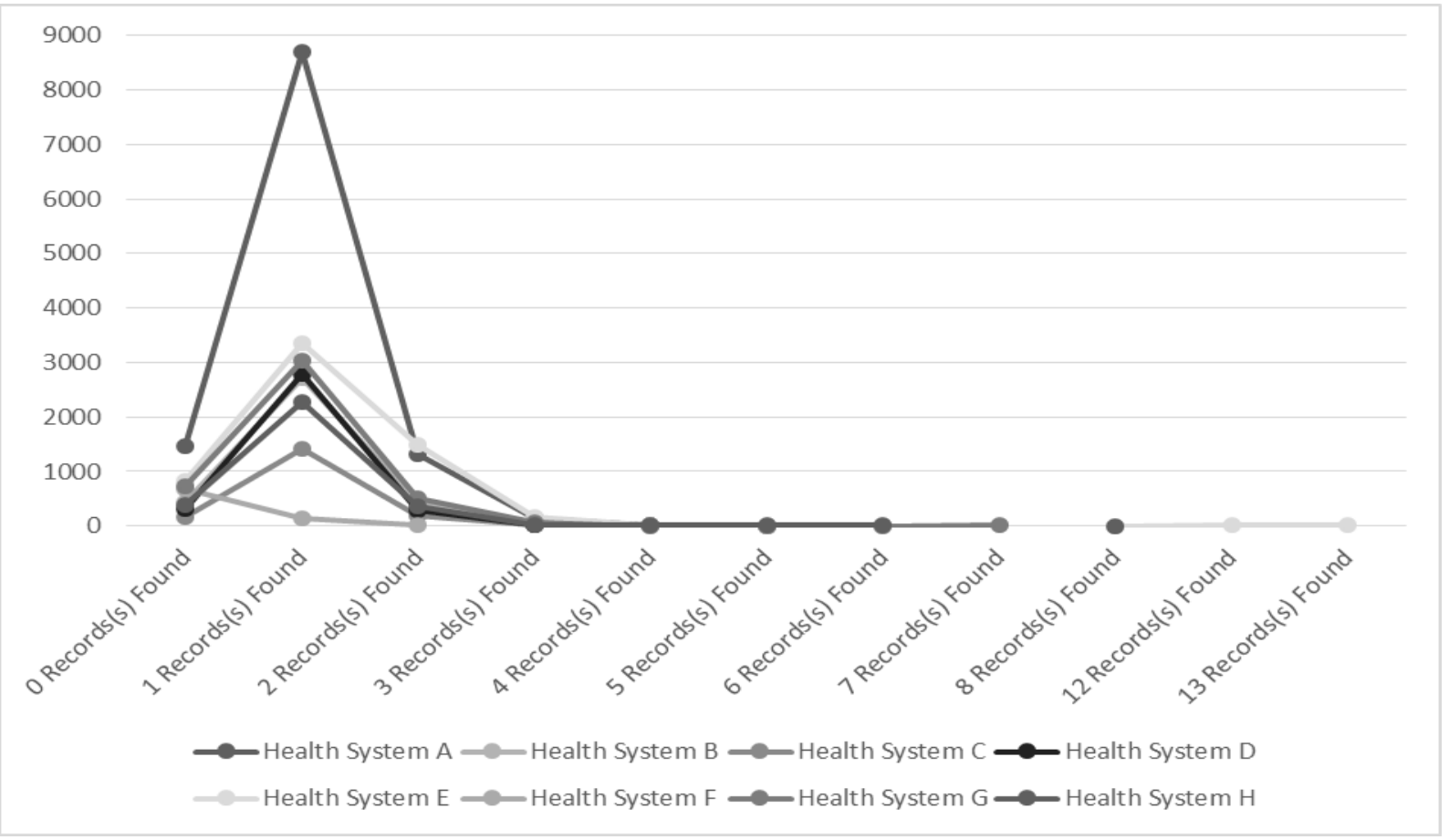

Figure 6: MIIC CDSi Query Results by Health Systems*, Week of 10/12/15 - 10/18/15 *Graph does not include data from Health System I, an outlier with much higher volume of queries 


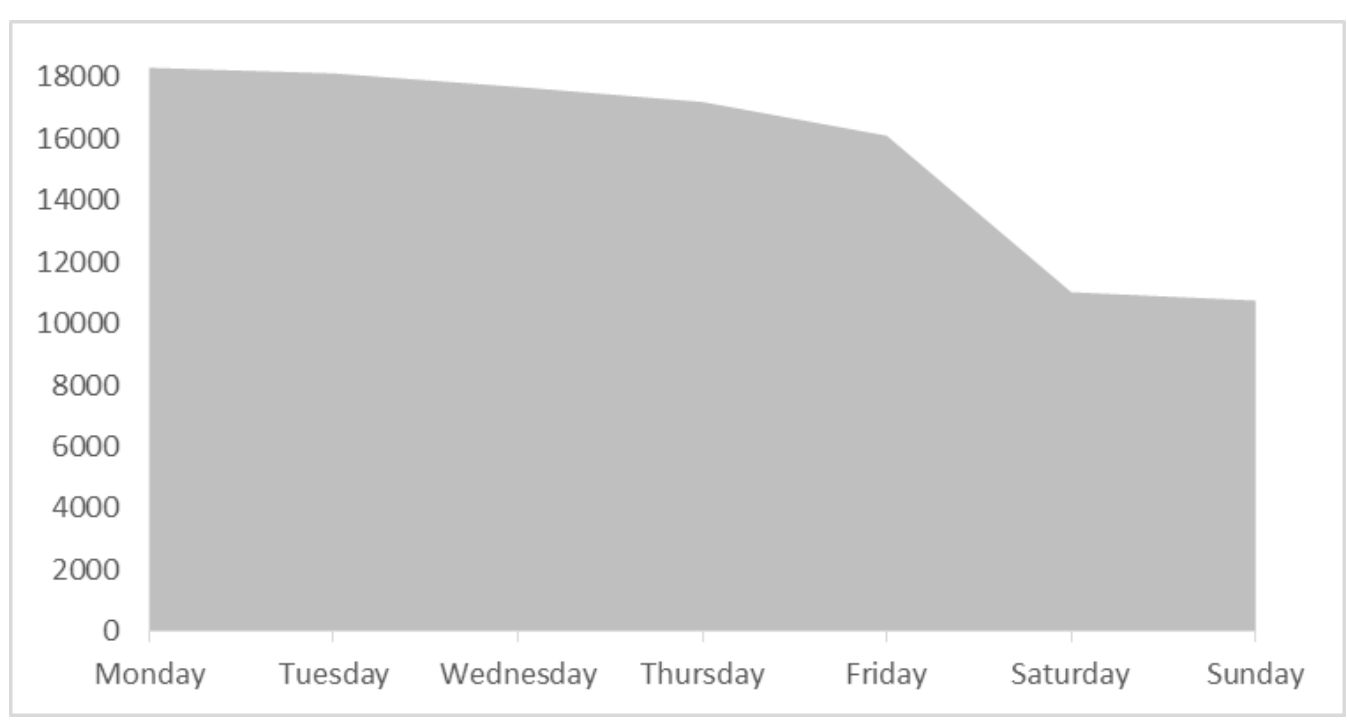

Figure 7: MIIC CDSi Query Results by Day, Week of 10/12/15 - 10/18/15

Table 4: MIIC CDSi Query Results by Local Public Health, Week of 10/12/15 - 10/18/15

\begin{tabular}{|c|c|c|c|c|c|c|c|c|c|}
\hline \multirow{2}{*}{$\begin{array}{l}\text { Queries by } \\
\text { Local Public Health }\end{array}$} & \multicolumn{8}{|c|}{ Number of Records Found } & \multirow[b]{2}{*}{ Total } \\
\hline & $\mathbf{0}$ & 1 & 2 & 3 & 4 & 5 & 6 & $\begin{array}{l}\text { Error / } \\
\text { Blank } \\
\end{array}$ & \\
\hline Local Public Health A & & 4 & & & & & & & 4 \\
\hline Local Public Health B & & 18 & 5 & & & & & & 23 \\
\hline Local Public Health C & & 6 & & & & & & & 6 \\
\hline Local Public Health D & & 11 & 1 & & & & & & 12 \\
\hline Local Public Health E & & 2 & 1 & & & & & & 3 \\
\hline Local Public Health F & & 56 & 4 & & & & & & 60 \\
\hline \multicolumn{10}{|l|}{ Local Public Health G } \\
\hline Local Public Health $\mathrm{H}$ & & 2 & 2 & & & & & & 4 \\
\hline Local Public Health I & 30 & 424 & 91 & 1 & & & 3 & 32 & 581 \\
\hline Local Public Health J & & 35 & 4 & & 3 & & & & 42 \\
\hline \multicolumn{10}{|l|}{ Local Public Health K } \\
\hline Local Public Health L & & 27 & 3 & & & & & 1 & 31 \\
\hline \multicolumn{10}{|l|}{ Local Public Health M } \\
\hline Local Public Health N & & 5 & & & & & & & 5 \\
\hline \multicolumn{10}{|l|}{ Local Public Health O } \\
\hline Local Public Health P & 1 & 43 & 8 & & & & & 9 & 61 \\
\hline
\end{tabular}




\section{Discussion}

The organizations that had implemented the Alternate Access functionality are using EHR technology which are considered mature products in the marketplace. The two EHRs with AA functionality are also products with high adoption rates in Minnesota and reflective of current EHR product landscape in Minnesota. The prevalence of affiliated sites with as much as 110 sites accessing via one AA installation poses both opportunities and challenges. It poses a perfect platform for rapid adoption of technology, but presents a complex scenario to track MIIC CDSi access by individual clinical sites. The queries to access MIIC were evenly distributed across the study time period with volume ranging from 1000 to 12,000 per week depending on size of the organization. The large volume of queries by a single organization needs additional research to understand the usage. Access is more on week days (Monday-Friday) and corresponds to access of MIIC for immunizations (one of preventive services) by primary care and local public health clinics. The access volume on week-ends, though less as compared to week days, may be an indicator for querying MIIC during urgent and emergency care visits.

The number of records located through access/queries is an indicator of the quality of data in IIS (MIIC in this study) and robustness of query parameters. The query yielded a single record for two-thirds of access and represents good quality data for $59 \%$ of searches. But one third resulted in error or blank response, which could be due to technical issues or lack of matching records and requires additional research. Five percent of searches did not yield any match and points to missing records in MIIC. Similarly, the numerous matches (close to 13 for some searches) may be an indicator of not so robust search criteria or the need to improve quality of data. The variability of records found across healthcare systems could be based on population they serve with healthcare spread across organizations and an indirect indicator of quality of various streams of immunization reporting.

\section{Conclusion}

This study analyzed usage patterns over a time period of 9 months (January-September 2015) and described use in depth for 1 week (October 12-18, 2015). There may be variability in other time segments, but review of data shows similar patterns across weeks, with the exception of an outlier organization. Follow-up study could yield insights into variability (if any) across organizations that present 'read-only' data view as compared to those which integrate data into their EHRs.

This research presents important aspects on access of MIIC CDSi, in terms of organizations, their affiliated sites, EHR technology in use, volume of queries, and records located. These offer insights into bi-directional exchange across IIS and EHRs. In addition, it highlights the number of records found through this access which is an indicator of quality of data, in particular comprehensiveness and accuracy. High EHR adoption in Minnesota and market dominance of select products offers an opportunity to promote use of MIIC CDSi through EHRs with better workflow integration.

Outreach to users/stakeholders with varying organizational roles (clinical, managerial and technical) will be needed to understand the numerous factors which impact IIS CDSi access and its use. Understanding the context of this use is critical to improve its access at point of clinical care and further studies are needed. These are much needed next steps for MIIC and overall IIS community as they plan provider outreach, prioritize their system enhancements to support bi- 
directional communications for Stage $3 \mathrm{MU}$ and other initiatives, and execute projects to improve population-level immunization rates.

Study Limitations. Study has some limitations, one of which is focus on a single IIS. Follow-up research needs to be conducted which looks into adoption and use of similar functionality supported by other IIS. Analysis did not track use at individual clinic level and how the queries were being generated. This could explain the volume of queries by site, day and outliers. Provider access and use at point of care is essential to impact immunization services, which may be influenced by organizational processes and technical factors. Additional research is needed to understand provider use, organizational and technical factors which impact use of IIS CDSi.

\section{Acknowledgements}

The authors would like to thank Erin Roche, manager of Minnesota Immunization Information Connection (MIIC) and Karen Soderberg, Assessment Lead at MDH Office of Health Information Technology for their support of this project. This project was supported by On the Horizon Grant from the University of Minnesota Informatics Institute (UMII).

\section{References}

1. U.S. Department of Health and Human Services. Office of Disease Prevention and Health Promotion. Healthy People 2020. 2010. Available at: http://www.healthypeople.gov/2020/topics-objectives/topic/immunization-and-infectiousdiseases. Accessed November 30, 2015.

2. Community Preventive Services Task Force. 2015. Recommendation for Use of Immunization Information Systems to Increase Vaccination Rates. J Public Health Manag Pract. (21)3, 249-52. PubMed

3. Centers for Disease Control and Prevention. Immunization Information Systems (IIS). 2014. Available at: http://www.cdc.gov/vaccines/programs/iis/index.html. Accessed October 15, 2015.

4. Minnesota Immunization Information Connection. MIIC Resources. 2009. Available at: http://www.health.state.mn.us/divs/idepc/immunize/registry/index.html. Accessed October 25, 2015.

5. Minnesota eHealth Initiative. 2015 Interoperable Electronic Health Record Mandate. 2007. Available at: http://www.health.state.mn.us/e-health/hitimp/index.html Accessed October 28, 2015.

6. Minnesota Department of Health. Minnesota's Health Reform Law. 2008. Available at: http://www.health.state.mn.us/healthreform/about/. Accessed September 29, 2015.

7. Minnesota DHS and MDH. Minnesota Accountable Health Model - State Innovation Model Grant. Partnership by the Minnesota Department of Human Services and Minnesota Department of Health. 2013. Available at: http://www.dhs.state.mn.us/sim. Accessed October 25, 2015.

8. Centers for Medicare and Medicaid Services. EHR Incentive Programs. 2010. Available at: http://www.cms.gov/ehrincentiveprograms. Accessed October 27, 2015. 
9. Advisory Committee on Immunization Practices (ACIP). Immunization Schedules. 2012. Available at: http://www.cdc.gov/vaccines/schedules/hcp/index.html. Accessed November 30, 2015.

10. Dixon BE, Gamache RE, Grannis SJ. 2013. Towards public health decision support: a systematic review of bidirectional communication approaches. Journal of the American Medical Informatics Association: JAMIA. 20(3), 577-583. Epub 03 2013. PubMed http://dx.doi.org/10.1136/amiajnl-2012-001514

11. Gamache R, Stevens KC, Merriwether R, Dixon BE, Grannis S. 2010. Development and Assessment of a Public Health Alert Delivered through a Community Health Information Exchange. Online $J$ Public Health Inform. 2(2). Epub 01 2010. PubMed http://dx.doi.org/10.5210/ojphi.v2i2.3214

12. Stevens LA, Palma JP, Pandher KK, Longhurst CA. 2013. Immunization registries in the EMR Era. Online J Public Health Inform. 5(2), 211-211. $\underline{\text { PubMed }}$ http://dx.doi.org/10.5210/ojphi.v5i2.4696

13. Rajamani S, Roche E, Soderberg K, Bieringer A. 2014. Technological and Organizational Context around Immunization Reporting and Interoperability in Minnesota. Online J Public Health Inform. 6(3). PubMed http://dx.doi.org/10.5210/ojphi.v6i3.5587

14. Agency for Healthcare Research and Quality (AHRQ). DEVISE: Data Exchange of Vaccine Information between an Immunization Information System and Electronic Health Record (New York). 2013. Available at: http://healthit.ahrq.gov/ahrq-funded-projects/devise-dataexchange-vaccine-information-between-immunization-information. Accessed

15. Dombkowski KJC. 2012. S. J. Redefining meaningful use: achieving interoperability with immunization registries. Am J Prev Med. 42(4), e33-35. Epub 03 2012. PubMed http://dx.doi.org/10.1016/j.amepre.2012.01.009

16. Consulting HLN. IIS and EHR Feature Overlap. 2014. Available at: https://www.hln.com/assets/pdf/HLN-IIS-EHR-Overlap-White-Paper.pdf. Accessed October 8, 2014.

17. U.S. Department of Health \& Human Services. Medicare and Medicaid Programs; Electronic Health Record Incentive Program-Stage 3 and Modifications to Meaningful Use in 2015 Through 2017; Final Rule 2015. Available at: http://www.gpo.gov/fdsys/pkg/FR-2015-1016/pdf/2015-25595.pdf. Accessed November 30, 2015.

18. U.S. Department of Health \& Human Services. The Affordable Care Act and Immunization. 2012. Available at: http://www.hhs.gov/healthcare/facts-and-features/fact-sheets/aca-andimmunization/index.html. Accessed November 30, 2015.

19. MDH Office of Health Information Technology. Clinics: Adoption and Use of EHRs and Exchange of Health Information, 2014. Available at: http://www.health.state.mn.us/ehealth/summaries/reportclinic2014.pdf. Accessed September 9, 2015.

20. MDH Office of Health Information Technology. Local Public Health: e-Health Capacity, Capability, and Challenges, 2014. Available at: http://www.health.state.mn.us/ehealth/summaries/reportlph2014.pdf. Accessed September 9, 2015. 